\title{
Introduction to the Special Edition on Engineering and Animal Ethics
}

\author{
Clare Palmer ${ }^{1} \cdot$ Gary Varner ${ }^{1}$
}

Accepted: 10 January 2018/Published online: 9 February 2018

(C) Springer Science+Business Media B.V., part of Springer Nature 2018

This special edition of the Journal of Agricultural and Environmental Ethics explores various effects that human engineering has, or could have, on the lives of non-human animals. The papers are the fruit of the Second Annual Bovay Workshop on Applied Ethics held at Texas A\&M University (TAMU) on March 28 and 29, 2017. The workshop was primarily sponsored by the Sue and Harry Bovay Professorship in the History and Ethics of Professional Engineering, a position currently held by Martin Peterson (Department of Philosophy, TAMU). Additional, logistical support was provided by the staff of the TAMU Department of Philosophy, and a portion of the travel funding for participants was contributed by Clare Palmer's Cornerstone Faculty Fellowship in TAMU's College of Liberal Arts.

For the 2017 Bovay Workshop on Applied Ethics, we proposed the topic: "Engineering and Animal Ethics." We initially wondered whether such a topic might be too narrow and specialized. The more we reflected on the idea, however, the broader we realized the possible scope for papers could be, because human engineering projects are so diverse, and they affect animals in so many different ways.

Historically, perhaps the most important way in which engineering projects have impacted animals has been through large construction projects in the natural environment, for instance the creation of roads, bridges, railways, pipelines, oil rigs, and dams. These can affect animals both directly-by fragmenting, destroying, or creating new wild animal habitats - and indirectly, by expanding and shaping the direction of human settlements in the landscape. Although animals are not the direct

Clare Palmer

c.palmer@tamu.edu

Gary Varner

g-varner@tamu.edu

1 Department of Philosophy, Texas A\&M University, College Station, TX 77843-4237, USA 
targets of human engineering in such cases, if we accept that at least some animals are of moral significance, the side-effects of these projects on animals, especially where they cause displacement, pain, or death, clearly raise ethical concerns.

However, such large-scale, landscape-level engineering projects are only one of the multitude of ways in which animals are now affected by engineering. In many current and emerging engineering projects, impacts on animals are not unintended side effects, but the goal of the intervention. Many projects aim to better manage and control animals' environments, in particular the environments of agricultural animals, sometimes with the goal of improving animal welfare. A well-known example here is Temple Grandin's work to re-engineer slaughterhouse access points in ways that are less stressful for the cattle entering them. Other projects aim to engineer the animals themselves in some way. Most obviously, this includes genetic engineering; but other forms of engineering animals involve the use of, for instance, electronic chips, engineered drugs, and (as in the human case) prostheses and other engineered medical appliances. Some of these "engineering solutions" — such as the veterinary ones - may have the goal of improving animal welfare. Others, though, are primarily intended for human benefit, and may raise concerns about increasing animal suffering (either by increasing the number of animals suffering or by increasing the intensity of their suffering), or about restricting animals' ability to perform natural behaviors.

In addition, it seems likely that we are in the very early days in terms of manipulating animals' environments — and animals themselves - through the use of engineering. Animals in homes, laboratories, and agriculture are likely to see their environments and their bodies ever more shaped and controlled by human engineering. Even some "wild" animals and their habitats are likely to be engineered, for instance as a contribution to human attempts to protect species against multiple anthropogenic extinction threats. In the near future, not only the animals, but some of the products we keep and kill them for-especially meat, milk, and cheese-are likely to be engineered too. But what will these forms of engineering mean for animal welfare? And what ethical questions are raised by the extension of engineering into virtually every aspect of animals' lives, behaviors, habitats, and even their secretions?

We hoped that the workshop on "Engineering and Animal Ethics" would provide an opportunity to explore these questions, and would, in particular, stimulate papers that considered ethical questions raised by novel engineering developments that are likely to impact the lives (and deaths) of so many animals. As we hope this collection shows, the workshop certainly succeeded in this aim. The eight papers included here are highly diverse, and include ethical discussions of engineering animals' environments, engineering animals' bodies, and creating engineered animal products. ${ }^{1}$ All these forms of engineering have, as the authors point out, impacts on animals' welfare; some have as their goal actually improving welfare

\footnotetext{
1 The papers in this edition were all refereed after the workshop. Each paper was double-blind refereed by one external reviewer, and also refereed by one internal referee who was present at the workshop. The author does not know the identity of the internal reviewer. Several papers went back to reviewers more than once. One paper was not given at the workshop but was contributed by a commentator from the workshop.
} 
(although whether they are likely to succeed in this goal may in part depend on what welfare is taken to mean); and some raise other ethical questions about, for instance, animals' integrity or wildness.

This special edition begins with two papers that, as it were, set out the lay of the land in the key areas in which engineering is impacting, or is likely to impact, animals.

In the first essay, Candace Croney (who is Director of the Center for Animal Welfare Science at Purdue University) and co-authors provide an overview of the different ways that engineering can be used to address welfare issues in the context of modern, production animal agriculture. They discuss various current examples of engineering environments to better suit the animals, of using both traditional selective breeding and genetic engineering to better fit the animals to their environments, and removing the animals from meat production using laboratorygrown meat. Croney et al. emphasize that agricultural animal welfare is what is sometimes called "a wicked problem," meaning that there usually is no solution that simultaneously addresses all of the related ethical concerns. Due both to continued human population growth and the continued spread of affluence, global demand for meat is likely to rise into the foreseeable future. That means, Croney et al. maintain, that engineering has a vital role to play in increasing the production of animal protein, but at the same time, affluent societies are expressing growing concerns about animal welfare as well as food safety and environmental impacts of animal agriculture.

The second essay, by Adam Shriver and Emilie McConnachie, provides a general argument for pursuing genetic engineering approaches to improving welfare when (as Croney et al. note) there is going to be widespread, continued reliance on intensive, confinement animal agriculture. They discuss various lines of current research that aim to use genetic engineering to improve animal welfare, arguing that a future of genetically engineered animals would be an improvement, morally speaking, over the status quo. They then reply to objections based on nonconsequentialist moral thinking and on comparisons to other alternatives to the status quo, such as reliance on plant-based alternatives to animal products and on in vitro-grown meat. Finally, they close by giving reasons for thinking that gene editing for welfare will remain relevant in the foreseeable future, because agricultural research centers are starting to treat gene editing as "the future of agriculture," gene editing is already extensively used to develop animal models for human diseases, and it can be used both to mitigate environmental impacts of agriculture and improve human nutrition.

The next paper, by Ian Werkheiser, discusses a currently available technological innovation called "Precision Livestock Farming" (PLF). This involves using various recent engineering advances to improve the monitoring and care of animals on large production operations. These include combinations of electronic chips, cameras, and other hardware as well as software that together allow farmers to monitor individual animals and to respond to their needs. For instance, PLF systems can measure out animals' food individually, take regular health readings, check barn temperatures, and so on. This, it might be argued, offers agricultural animals something like the care that a traditional farmer, who knows his or her animals 
individually, might be able to offer, but at the large scales that are increasingly the norm in animal agriculture. So, the use of PLF may suggest that even as farms scale up in size, agricultural animal welfare need not decline, and could improve, while animal agriculture might also be made more sustainable and more profitable using PLF. However, as Werkhauser points out, while there may be improvements in some of these ways, PLF still raises a variety of ethical concerns. Werkheiser focuses on the potential loss of identity for farmers, who no longer have a personal relationship with individual animals, which (he suggests) might be regarded as constitutive of being a livestock farmer. And, for their part, animals might also lose their individual identities. For while the technologies of precision livestock farming may be able to identify and differentiate each animal's needs, that is very different from being known personally as an individual, and engaging in an interactive relationship with a particular farmer.

Another type of currently available engineering project is discussed in the next paper by Clare Palmer, Gervi Pederson, and Peter Sandøe. Their focus is the use of non-surgical means of engineering animals' fertility. As the authors point out, fertility control drugs can have multiple uses. Most prominently, they can make animals easier to handle and to live with by changing animals' behavior, which is particularly important in companion and agricultural animals; and they can control increases in population size, which is primarily important in managing populations of wild and feral animals. Given this, the goal of fertility control is not normally animal welfare directly. But the question that explicitly runs through this paper is whether non-surgical fertility control technologies do better, in terms of animal welfare, than existing or likely future alternatives for managing populations of animals or their behaviors. If, as the title of the paper suggests, the most plausible alternatives are castration or culling, it's at least worth asking whether fertility control might promise better outcomes in terms of welfare, even if it raises other ethical questions, for instance about rights to reproduction or loss of wildness.

The remaining four papers focus, in several different ways, on the use of genetic engineering to address welfare issues. In his paper, Marcus Schultz-Bergin focuses on a specific new gene editing technique, CRISPR. Schultz-Bergin argues that CRISPR has the potential to be a game-changer, not only scientifically, but also in ethical terms. This is because the technique allows for gene editing without requiring transgenesis - the insertion of genetic material from another organism. Transgenesis, especially between species, has been the focus of many "in principle" objections to genetic modification. So, if genes can be edited without transgenesis, Schultz-Bergin argues, one of the major ethical objections to genetic engineering can be bypassed. This doesn't, of course, mean that no ethical concerns remain. The use of CRISPR on animals, in particular, still raises animal welfare questions. As Schultz-Bergin argues, although CRISPR may be a relatively accurate form of gene editing, there may still be off-target effects with implications for animal welfare. And while some applications of CRISPR may ultimately improve animal welfare (for instance, allowing for cattle to be born polled, rather than having their horns painfully removed), it is also likely to increase the number of sentient animals used in experimentation. So, while the technique may not generate some traditional kinds 
of ethical concerns about genetic engineering, Schultz-Bergin concludes, it's likely to intensify others.

The paper by Nicolas Delon and Duncan Purves also focuses on the use of gene editing techniques such as CRISPR. However, their concern is with a very specific kind of gene editing: large-scale engineering projects to adapt wild animals in order to reduce the prevalence of wild animal suffering. Delon and Purves accept that wild animal suffering is immense, and they argue that if we think that all of that suffering is intrinsically bad, then we have a (defeasible) obligation to try to reduce it. One way that has been proposed for doing so is by using gene editing on a large scale to (for instance) reduce the very large number of offspring that are produced by " $r$ strategist" species, so that fewer of their offspring would have very short lives that are not worth living; or to reprogram predators, so that their predatory traits are repressed. However, Delon and Purves argue that such uses of gene editing to promote animal welfare in any near future are unacceptable, because the wild context is so complex and the cascading effects of such large-scale interventions are currently extremely hard to predict. Thus, we have no way of knowing at present whether such large-scale engineering interventions actually would reduce animal suffering at all. While they leave open the possibility that at some point in the future we may have metrics and models that can give us a better idea of the impacts of such interventions, for the time being we should not pursue any large-scale engineering project of this kind in the wild.

Next, Josh Milburn's paper discusses the ethics of "clean milk," meaning "dairy products created by biotechnological means, without the need for cows." There has been much discussion among animal activists of "clean meat," but, Milburn says, no discussion of clean milk. He notes, however, that a company currently claims to be ready to produce milk that is virtually indistinguishable from cows' milk using a fermentation process. Instead of culturing cells taken from live animals, as is currently being done with clean meat, the process would involve genetically modifying yeast to ferment plant-based sugars into the whey and proteins found in cows' milk, and finishing the product off by adding some plant-based fats and other ingredients. While animal activists have raised various concerns about clean meat, Milburn argues that "animal ethicists and activists should embrace" clean milk, "it is a product about which they should have few, if any, ethical qualms." His analysis includes responses to arguments from various concerns that animal activists have raised about clean meat or scavenged meat, for instance that consuming it represents sentient animals as mere resources.

Finally, Tatjana Višak uses a hypothetical future use of genetic engineering to focus on a philosophical question in population ethics. Višak compares what she calls "the mundane practice," in which current breeds of agricultural animals are raised humanely and slaughtered painlessly at an age well short of their life expectancy at birth, to what she calls "the engineering practice," in which animals are genetically engineered to have short life expectancies and to "drop down dead" at precisely the age that current animals are slaughtered. Višak's intuitive judgment-which she thinks is widely shared among animal ethicists-is that the two practices would be equally wrong. In her paper, she explains how current theories in the literature of population ethics fail to justify that intuitive judgment 
about what are called "non-identity cases." These are cases in which two possible future populations are compared, but the individuals involved differ in fundamental ways, which would include having been engineered to have a short life expectancy. Višak notes that in the choice between adopting a larger dog or smaller one, people generally don't take the fact that smaller dogs tend to live longer as determinative, but she suggests that choosing to engineer animals with shorter life expectancies is different. The reason, she suggests, is that we think ourselves unable to do anything about the larger dog breeds' shorter life expectancies, whereas applying genetic engineering to shorten an agricultural breed's life expectancy is very much doing something about it.

Those are the eight papers that resulted from the Second Annual Bovay Workshop on Applied Ethics at Texas A\&M. We thank the Sue and Harry Bovay endowment in engineering ethics, the College of Liberal Arts, and the Department of Philosophy at Texas A\&M for making the workshop, and publication of the papers in this special edition, possible. We hope that the work fostered by the workshop will stimulate more consideration of the role of engineering in present and future developments in animal ethics. 